\title{
SERUM GLUTAMIC OXALACETIC TRANSAMINASE LEVELS IN DIPHTHERITIC MYOCARDITIS
}

\author{
BY \\ E. CHESLER \\ From the Johannesburg Fever Hospital, Johannesburg \\ Received July 29, 1957
}

Serum glutamic oxalacetic transaminase (S.G.O.T.) is normally present in human serum, skeletal muscle, brain, liver, kidney, testis, and lung. Its highest concentration, however, is in cardiac muscle. Elevated S.G.O.T. levels occur with active dissolution of the tissues containing this enzyme and abnormally high levels are associated with cardiac cellular necrosis. This elevation is directly related to the extent of tissue destruction (LaDue et al., 1954; LaDue and Wroblewski, 1955; Nydick et al., 1955).

In the cases presented here, serial S.G.O.T. levels estimated during the course of severe diphtheria are compared with concomitant electrocardiograms.

\section{CASE REPORTS}

Case 1. An 8-year-old boy was admitted with the complaint of sore throat for six days and difficulty in breathing for one day. Examination showed an extremely ill boy with laryngeal stridor, sternal recession, and obvious "bull-neck" lymphadenopathy. The tonsils, uvula, and pharynx were covered with diphtheritic membrane. The remainder of the physical examination was negative. 120,000 units of anti-diphtheritic serum was given intramuscularly and a tracheotomy performed; intramuscular penicillin and oral prednisone

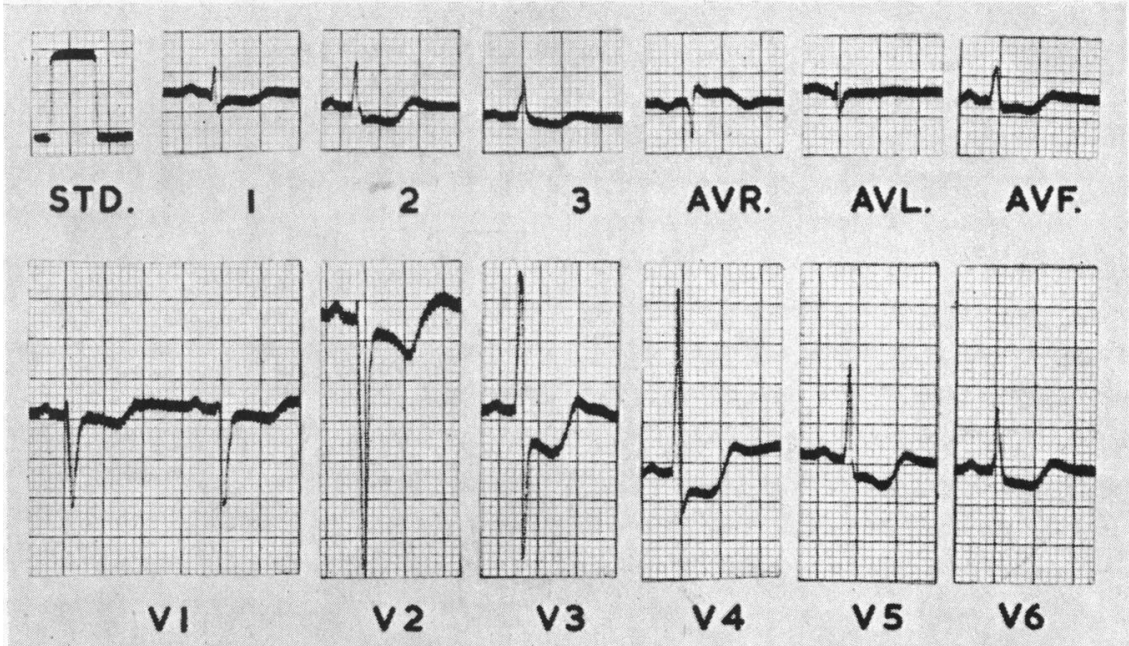

Fig. 1.-Cardiogram of Case 1, showing abnormal changes during the course of myocarditis. 


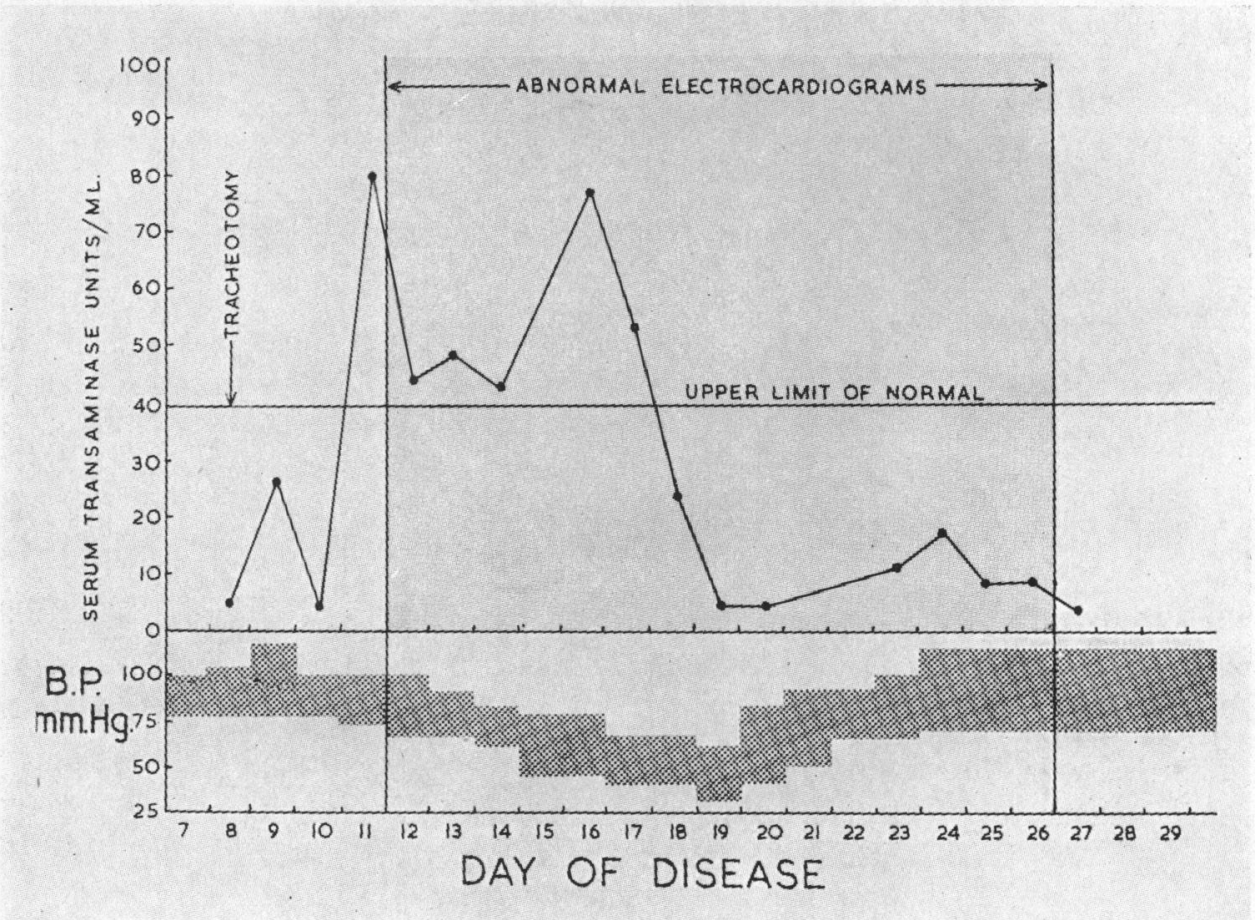

Fig. 2.-Diagram showing relationship of S.G.O.T. levels to cardiac changes in Case 1.

and oxytetracycline were given post-operatively. On the twelfth day of the disease he showed clinical evidence of carditis manifested by vomiting, marked increase in pallor, hypotension, and cardiac enlargement. Electrocardiographic abnormalities were also noted at this time (Fig. 1) and persisted for fourteen days. At no stage were there signs of congestive cardiac failure.

Laboratory Investigations. A virulent strain of $C$. diphtheriae was isolated from the throat swabs. Serial S.G.O.T. estimations are shown in Fig. 2. Electrocardiograms recorded on the seventh and ninth day of disease were normal. The cardiograms on the eleventh day when the S.G.O.T. level was 80 units per ml., showed doubtful depression of the S-T segments. Cardiograms from the twelfth day onwards revealed progressive depression of the S-T segments in epicardial leads, with reciprocal S-T elevation in cavity leads, and flattening or inversion of the T waves. Maximum depression of the S-T segment of $5 \mathrm{~mm}$. was noted on the sixteenth day following which there was a slow return to normal, and iso-electric S-T segments with upright $T$ waves were once again recorded on the 27 th day of the disease. On the sixteenth and the eighteenth days there was complete A-V dissociation. A representative cardiogram is shown in Fig. 1.

Case 2. A 7-year-old boy was admitted on the seventh day of his illness. Examination disclosed extreme toxæmia associated with a "bull-neck" lymphadenopathy, and membrane formation on the tonsils, uvula, and pharynx. The remainder of the physical examination was negative. He was given 50,000 units of anti-diphtheritic serum and penicillin and oxytetracycline orally. There was little improvement in his condition and on the ninth day of the disease he complained of persistent nausea, epigastric and substernal pain, and vomited repeatedly. His blood pressure dropped from $110 / 70$ to $80 / 60 \mathrm{~mm}$. $\mathrm{Hg}$ and cardiac enlargement was noted. From the tenth to the sixteenth day of the disease a reversed split of the pulmonary second sound was heard. By the eighteenth day of the disease he was asymptomatic and his heart size had returned to normal. At no stage was there evidence of congestive cardiac failure.

Laboratory Investigations. A throat swab yielded a virulent strain of diphtheria bacillus. Serial S.G.O.T. estimations are shown in Fig. 3. 


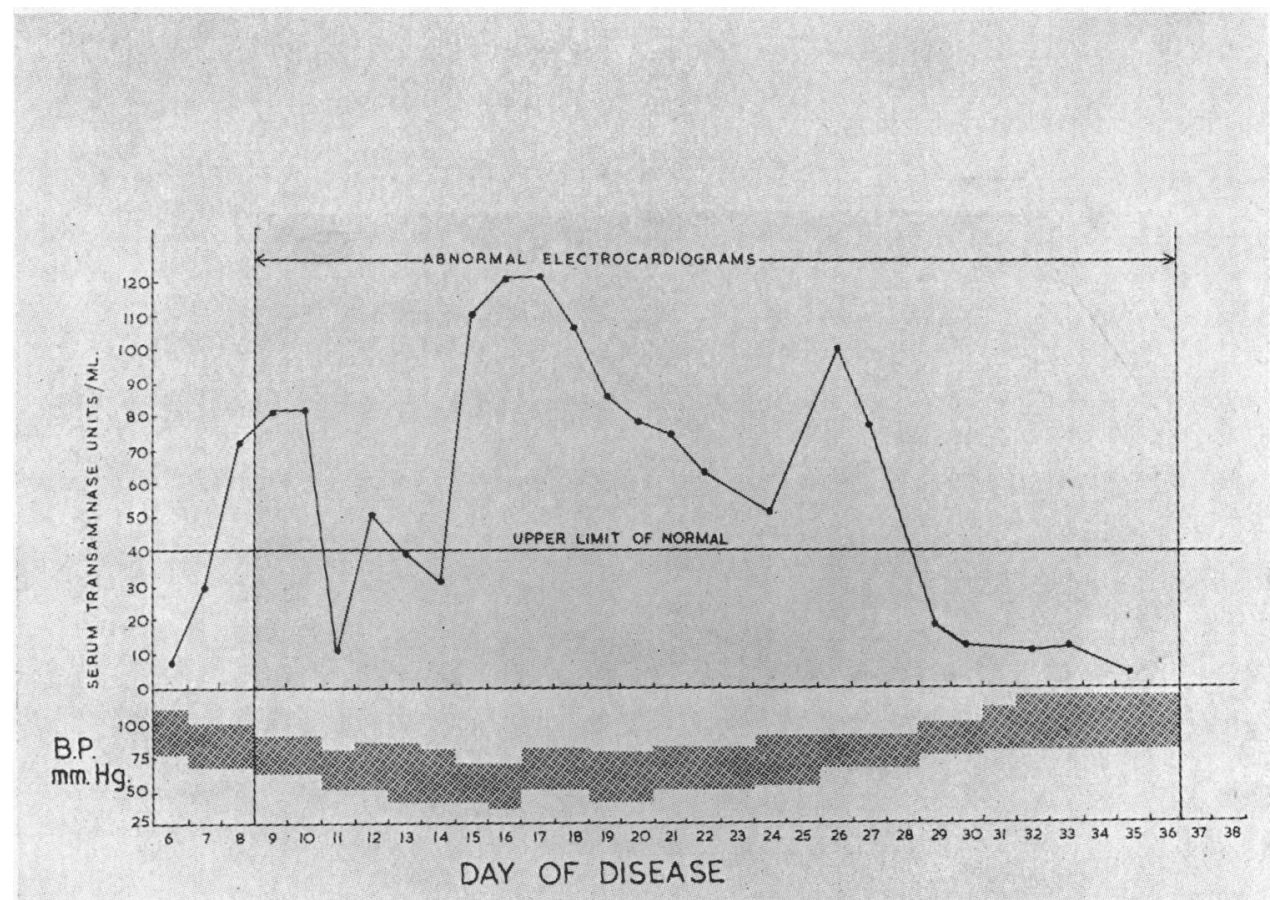

FIG. 3.-Diagram showing relationship of S.G.O.T. levels to cardiographic changes in Case 2.

Electrocardiograms. Day 5, 6 and 7: Normal.

Day 8: Sinus rhythm. Probable early depression of the S-T segments and flattening of the $T$ waves.

Day 9: Sinus rhythm; definite depression of the S-T segments in epicardial leads with S-T elevation in cavity leads. T waves flattened or inverted.

Day 10: Complete A-V dissociation and left bundle branch-block.

Day 11: Sinus rhythm. Grade 1 A-V block (P-R interval 0.24 sec.) and left bundle-branch block.

Day 12, 13 and 14 (Fig. 4a): Sinus rhythm with Grade 2 heart block, Wenckebach periods, and left bundle-branch block.

Day 15 (Fig. 4b) and 16: Complete A-V dissociation and left bundle-branch block. The elevated S-T segments usually recorded by right ventricular epicardial leads in left bundle-branch block were now markedly depressed in leads VI to V3: further depression of the S-T segments in leads V4 to V6 is also evident.

Day 17: Sinus rhythm. Grade $1 \mathrm{~A}-\mathrm{V}$ block (P-R interval $0.3 \mathrm{sec}$.). The abnormalities of the S-T segments and the $T$ waves were still evident, but the pattern of left bundle-branch block had disappeared.

From the 18th day onwards, a progressive return of the S-T segment to the iso-electric line and reversion of the $T$ waves to normal occurred. On the 37 th day of disease a completely normal cardiogram was recorded.

Case No. 3: A 7-year-old boy was admitted on the fourth day of his illness. He was critically ill, with laboured respiration as a result of membrane formation in the throat and nose with accompanying soft tissue cedema. Massive "bull-neck" lymphadenopathy was present. The blood pressure was $68 / 40 \mathrm{~mm}$. $\mathrm{Hg}$. The extremities were cold and cyanosed. There was no clinical evidence of carditis. 120,000 units of antidiphtheritic serum were given intramuscularly and a tracheotomy performed. In addition, he received penicillin, oxytetracycline, hydrocortisone, and nor-adrenaline. There was no response and he died in peripheral circulatory failure after 36 hours in hospital, on the 6 th day of the disease. Throat swabs yielded a virulent 
strain of diphtheria bacillus. On the fifth day of the disease the electrocardiogram was normal, and the S.G.O.T. was 20 units per $\mathrm{ml}$.

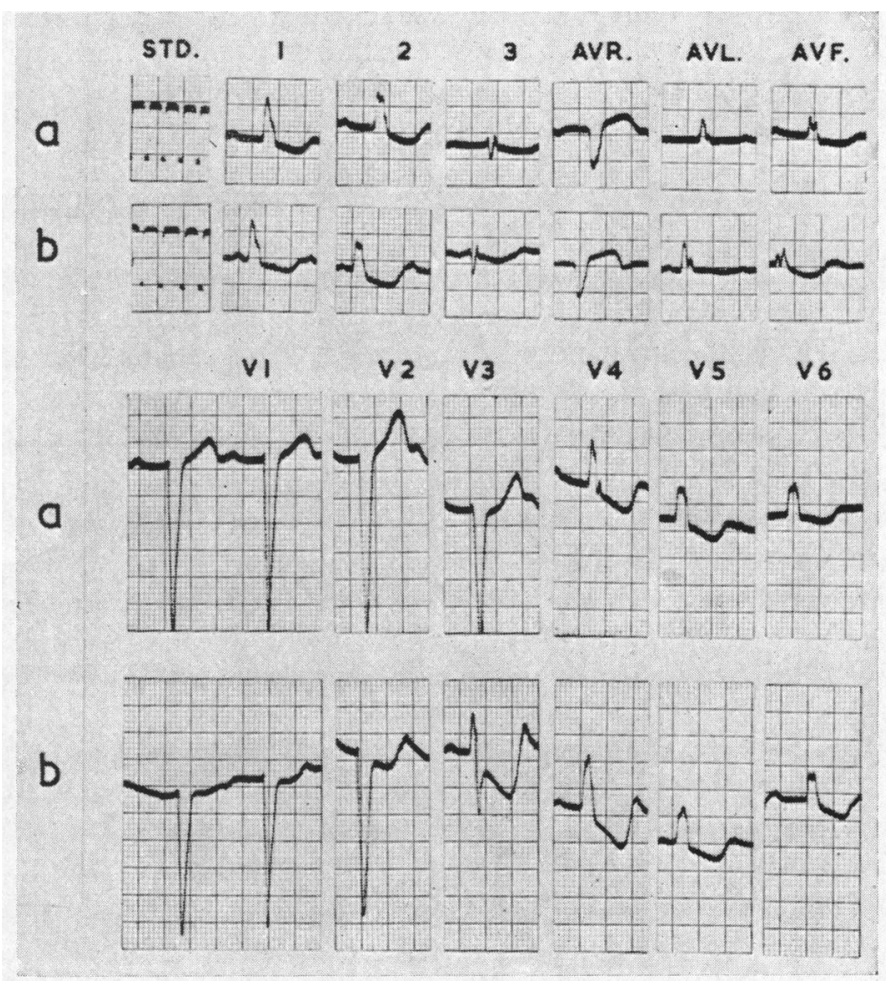

FIG. 4.-Cardiogram of Case 2, showing abnormal changes observed during the course of myocarditis on the fourteenth and the fifteenth days ( $a$ and $b$, respectively).

Case 4. A girl of 10 years was admitted on the twelfth day of her illness. Examination revealed a severe "bull-neck" diphtheria. She was given penicillin, oxytetracycline and prednisone. Throughout her stay in hospital, there was no evidence of myocarditis. Throat swabs yielded a virulent strain of C. diphtheriæ. The S.G.O.T. levels are shown below.

\begin{tabular}{lcccrrrrrrrrrrrrrrrrr} 
Day of disease &. &.. & 11 & 12 & 13 & 14 & 15 & 16 & 17 & 18 & 19 & 20 & 21 & 22 & 24 & 25 & 26 & 27 & 28 & 40 \\
\hline S.G.O.T. (units per $\mathrm{ml}$. ) &. & 5 & 5 & 5 & 5 & 9 & 14 & 28 & 27 & 21 & 7 & 5 & 23 & 61 & 24 & 41 & 24 & 5 & 6
\end{tabular}

Case 5. A girl, aged 3 years, was admitted on the fifth day of her illness. There was extensive membrane formation in the throat and larynx necessitating a tracheotomy shortly after admission. "Bull-neck" lymphadenopathy was present. She was treated with 40,000 units of anti-diphtheritic serum, penicillin, and oxytetracycline. At no stage in the disease was there clinical or cardiographic evidence of myocarditis. Throat swabs yielded a virulent strain of C. diphtheriæ. The S.G.O.T. levels are shown below.

\begin{tabular}{llllrrrrrrrrrrrrrrrrrr}
\hline Day of disease & $\ldots$ & $\ldots$ & $\ldots$ & 6 & 8 & 9 & 10 & 11 & 13 & 14 & 15 & 16 & 17 & 18 & 20 & 21 & 22 & 23 & 24 & 25 \\
\hline S.G.O.T. (units per ml.) & & $\ldots$ & 31 & 24 & 11 & 5 & 31 & 15 & 5 & 31 & 25 & 9 & 46 & 42 & 59 & 59 & 46 & 16 & 6 \\
\hline
\end{tabular}


Case 6. A boy, aged 2 years, suffering from "bull-neck" diphtheria was admitted on the sixth day of his illness. He was treated with penicillin, anti-diphtheritic serum, and oxytetracycline, but on the tenth day of disease he developed clinical evidence of myocarditis. Electrocardiogram showed left bundle-branch block, gross depression of the S-T segments in epicardial leads, with S-T elevation in cavity leads. Despite supportive therapy, he died three days later. S.G.O.T. estimations on the tenth and twelfth days were 88 and 220 units per ml. respectively.

\section{Discussion}

In diphtheria, clinical and cardiographic evidence of myocarditis is commonly observed during the second week of the disease (Wood, 1956). In the six cases presented here, serial S.G.O.T. estimations and daily cardiograms were performed during, and preceding this period. In Cases 1,2 , and 6, the clinical diagnosis of carditis was substantiated by cardiographic studies. In Cases 1 and 2, an elevated S.G.O.T. level was noted a day before the first unequivocally abnormal cardiogram and persisted during the period of worst carditis, returning to normal levels (below 40 units per ml.) several days before regression of cardiographic changes. In Case 2, after the onset of carditis, the sharp rise in the S.G.O.T. level on the 15th day (Fig. 3) coincided with cardiographic evidence of further cardiac damage at this time (Fig. 4). In Cases 3, 4, and 5, there was no clinical or cardiographic evidence of carditis, despite the early manifestations of severe toxæmia. In Case 3, where death occurred due to peripheral vascular failure, a normal S.G.O.T. of 20 units per ml. was obtained and the marked systemic effects of the diphtheria toxin in the absence of myocarditis, did not result in an elevated S.G.O.T. level. In Cases 4 and 5, there was a transient rise in the S.G.O.T. at about the 24 th and 18 th days respectively.

A constant feature of diphtheritic myocarditis is hyaline degeneration and necrosis of muscle fibres (Gore, 1948). The release of glutamic oxalacetic transaminase into the circulation from injured cardiac muscle provides an index of active cellular damage. Consequently, the early elevated levels may be expected to return to normal sooner than the cardiographic changes which continue to reflect abnormal patterns as a result of ensuing fibroblastic repair. The transient slight elevation of the S.G.O.T. levels in Cases 4 and 5 may be due to minimal myocardial damage insufficient to produce clinical and cardiographic abnormalities, and could be a pointer to the exercise of caution in the early ambulation of such cases.

Further study of the S.G.O.T. levels in diphtheritic myocarditis may demonstrate this test to be of considerable value in the early diagnosis of this condition. It may prove to be particularly useful in those cases where carditis is suspected clinically but cardiographic studies are inconclusive.

\section{SUMMARY}

Serial S.G.O.T. estimations with daily electrocardiograms were carried out in six cases of severe diphtheria. Three cases complicated by myocarditis showed abnormally elevated levels which correlated well with the cardiographic studies.

I am greatly obliged to Dr. A. L. Jackson, Superintendent of the Johannesburg Fever Hospital for permission to publish these cases. My thanks to the South African Institute for Medical Research for the laboratory investigations. I am indebted to Dr. R. M. Brueckner for permission to study one of her cases and to Dr. L. Schamroth for constant advice and helpful criticism.

\section{REFERENCES}

LaDue and Wroblewski, F. (1955). Circulation, 11, 871.

L- , and Karmen, A. (1954). Science, 120, 497.

Nydick, I., Wroblewski, F., and LaDue, J. S. (1955). Circulation, 12, 161.

Wood, P. (1956). Diseases of the Heart and Circulation. 2nd ed., Eyre and Spottiswoode, London.

Gore, I. (1948). Amer. J. med. Sci., 215, 257. 